\title{
The Development of A Large-Area Windowless Energy Dispersive X-ray Detector for STEM-EDX Analysis
}

\author{
K. Tamura ${ }^{1}$, R. Namekawa ${ }^{1}$, R. Buchhold ${ }^{2}$, B. Hammell ${ }^{2}$ and A. Sandborg ${ }^{2}$, T. Satoh ${ }^{3}$, M. Konno ${ }^{3}$,
} H. Inada ${ }^{1}$, K. Nakamura ${ }^{1}$, Y. Taniguchi ${ }^{1}$, T. Hashimoto ${ }^{1}$

1. Advanced Microscope System Design Dept., Hitachi High-Technologies Corporation, Ibaraki 312-8504, Japan

2. EDAX Inc., 91 McKee Drive Mahwah, NJ 07430

3. Global Application Center, Hitachi High-Technologies Corporation, Ibaraki 312-8504, Japan

Energy-dispersive X-ray spectrometry (EDX) is widely used for elemental analysis of nano meter scale areas with scanning transmission electron microscopes (STEMs). However, intensities of characteristic X-rays detected by conventional EDX detectors are not high enough to obtain x-ray mappings with higher definition and better signal to noise ratio in a shorter period of time. Increasing the probe current may worsen the spatial resolution of the elemental mapping, and longer acquisition time causes specimen damage by electron beam irradiation. Improvement of the X-ray detection sensitivity is therefore indispensable for high quality elemental mapping.

Recently, silicon drift detectors (SDDs) have become very popular as high throughput EDX detectors. High throughput X-ray detection is realized by low capacitance of a small collecting anode in SDD. Therefore, the SDD has efficient X-ray detecting capability with shorter dead time, compared with the Lithium- drifted silicon ( $\mathrm{Si}(\mathrm{Li})$ ) detector.

A highly sensitive STEM-EDX system based on the HD-2700 dedicated STEM [1] with an SDD detector with a sensor area of $100 \mathrm{~mm}^{2}$ has been developed. Optimizing the design of the collimator and the SDD positioning ensures an X-ray detection solid angle of $1.1 \mathrm{sr}$. and an elevation angle of $25.4 \mathrm{deg}$. for the HD-2700 standard pole piece model.

Moreover, we have adopted the windowless design to the new large-area EDX. The effectiveness of the windowless design has been examined [2] by using a $\mathrm{Si}$ (Li) detector. In the conventional EDX detector, an ultra-thin window (UTW) has been commonly used to improve low energy X-ray sensitivity. In such designs, the UTW is positioned in front of the X-ray detection element and the window material is reinforced with, for example, a Si grid to withstand the atmospheric pressure. Absorption of X-ray intensity by the window material and the associated reinforcement decreases the $\mathrm{X}$-ray signal detection efficiency. The windowless design enables improvement of detection efficiency by eliminating absorption of the window material and the associated reinforcement.

Figure 1 shows a comparison of 200keV STEM-EDX spectra of a SiN specimen measured with the large-area windowless EDX and the conventional UTW EDX (with a detection area size of $30 \mathrm{~mm}^{2}$ ). Though the nominal solid angle has been expanded 3 times, the signal intensities of the characteristic $\mathrm{X}$ rays detected with the former EDX is 10 times higher than that with the latter EDX at Nitrogen $\mathrm{K}$ line, and 8 times higher at Silicon $\mathrm{K}$ line. The relatively higher elevation angle is also advantageous to reduce absorption of lower energy $\mathrm{x}$ ray in the specimen.

EDX elemental maps and HAADF-STEM image of a Si device specimen are shown in Fig. 2. These maps and image were collected by the large-area windowless EDX in 5 minutes.

[1] H. Inada et al, J. Electron Microsc., 58 (2009), p.111-122.

[2] S. Isakozawa et al, J. Electron Microsc., 59(6), (2010) p. 469-472. 


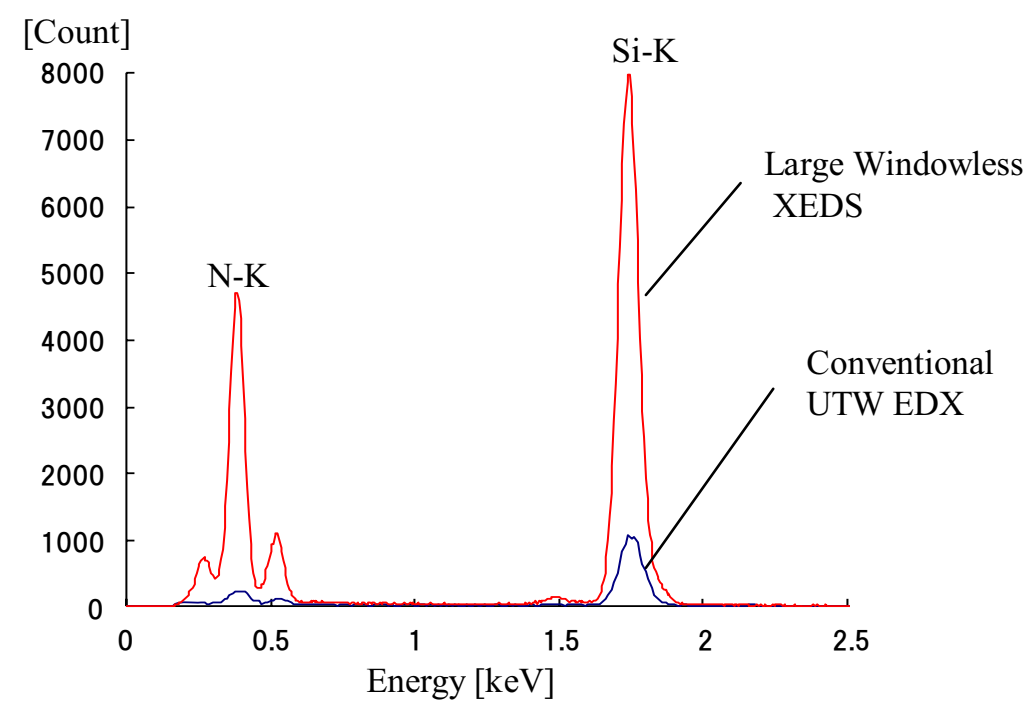

Figure 1. Comparison of $200 \mathrm{keV}$ STEM-EDX spectra of $\mathrm{SiN}$ specimen measured with the large windowless EDX and the conventional UTW EDX.
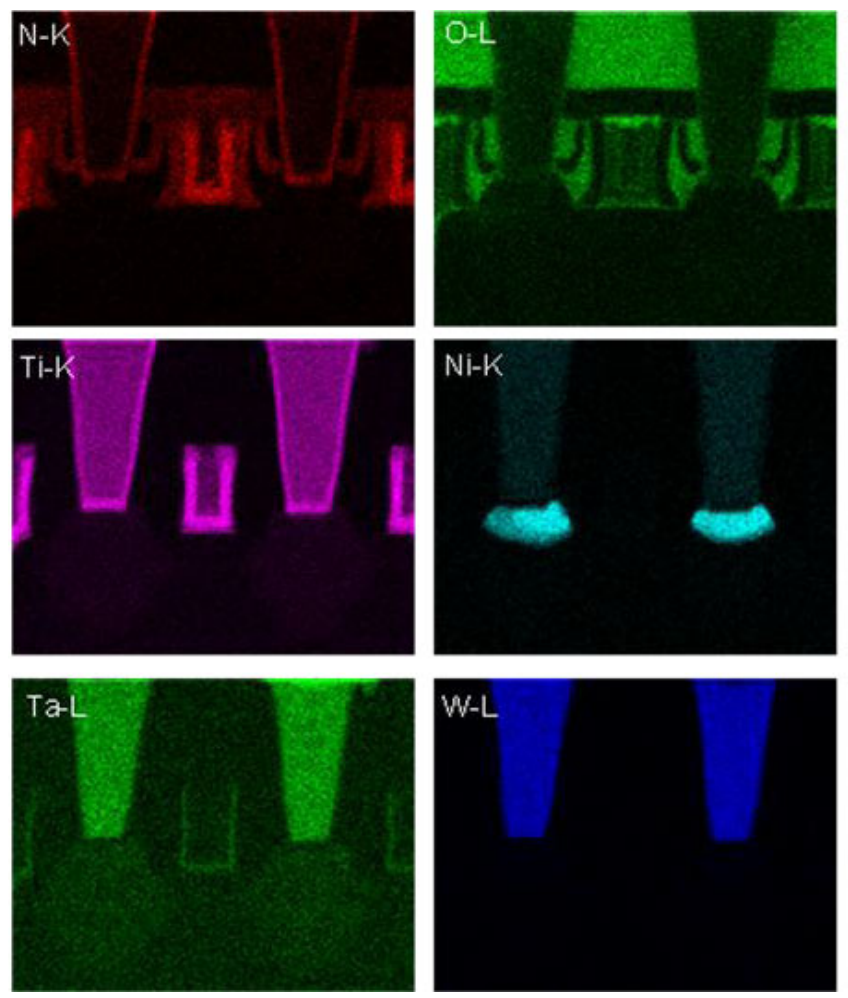
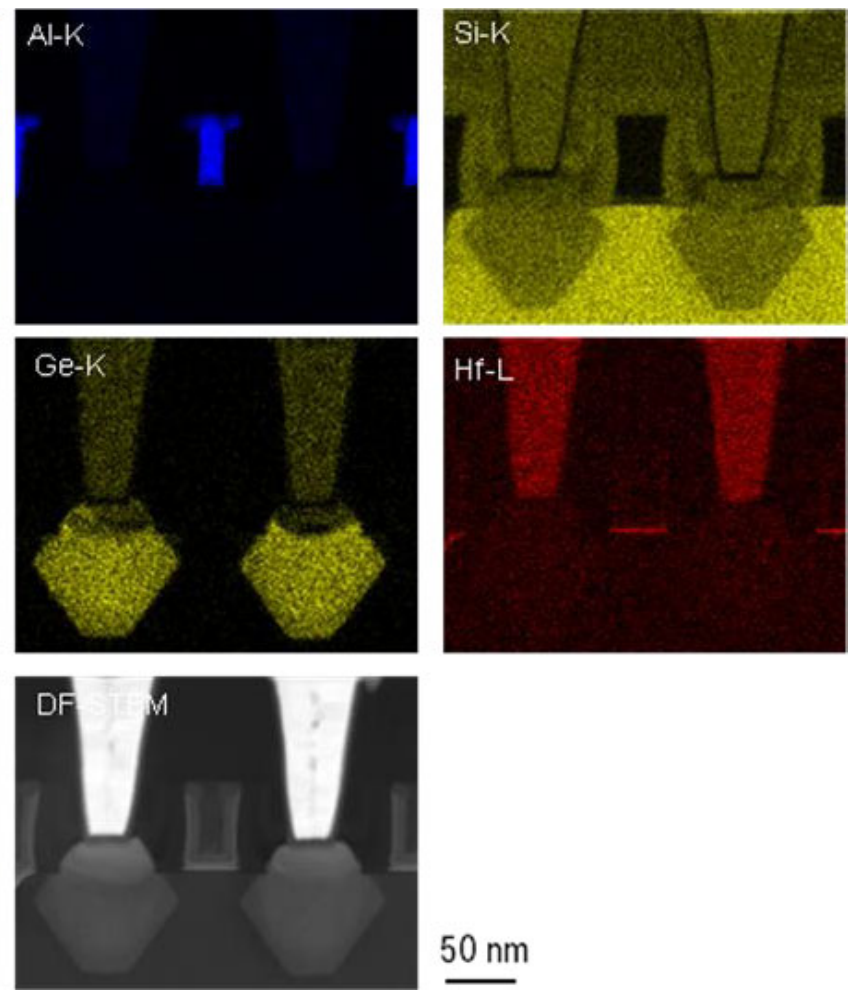

$\underline{50 \mathrm{~nm}}$

Figure 2. EDX elemental maps and HAADF-STEM image of a Si device specimen are collected by the large-area windowless EDX. The number of pixels for each map and the STEM image is $256 \times 200$, and the acquisition time is 5 minutes. 